\title{
Characteristics and risk factors for antituberculosis drug-induced liver injury in a cohort of patients with cirrhosis in a tertiary referral university teaching hospital in Thailand
}

\author{
Passisd Laoveeravat ${ }^{1, \#}$, Nicha Wongjarupong ${ }^{1,2, \#}$, Chonlada Phathong ${ }^{1}$, Cameron Hurst ${ }^{3}$, \\ Sombat Treeprasertsuk', Rungsun Rerknimitr', Roongruedee Chaiteerakij ${ }^{1, *}$
}

Abstract

Background: Cirrhotic patients are susceptible to drug toxicity, which presents frequently with antituberculosis drug (ATD) treatment. Previous studies of ATD-induced liver injury (ATDILI) in cirrhotics have been limited to patients with early-stage cirrhosis.

Objectives: To describe characteristics and determine risk factors for ATDILI in cirrhotic patients.

Methods: We included 64 cirrhotic patients treated with ATDs between 2006 and 2016 in a tertiary referral university teaching hospital in Bangkok, Thailand. Cirrhosis was diagnosed by radiological features, including small-sized nodular liver and/or caudate lobe hypertrophy or evidence of portal hypertension (collateral vessels, varices, and/or splenomegaly). Clinical information was retrospectively abstracted. Characteristics of patients with ATDILI vs. those without ATDILI were compared.

Results: Six (9.4\%) patients developed ATDILI with the median duration from ATD initiation of 14 days (range: 6-66). All the 6 patients who developed ATDILI received 3 hepatotoxic ATDs (isoniazid, rifampin, and pyrazinamide) and had Child-Turcotte-Pugh class B cirrhosis. The patients with ATDILI were found to have a higher percentage of human immunodeficiency virus (HIV) infection than patients without ATDILI ( $50 \%$ vs. 8.6\%; $P=0.02)$.

Conclusions: Cirrhotic patients, particularly those with underlying HIV infection, are at risk of developing ATDILI. Pyrazinamide should be used cautiously in cirrhotic patients due to the significantly increased risk of ATIDLI. This study supports the current recommendation for the use of ATD in patients with cirrhosis; however, the ATD regimen should be carefully selected, particularly for cirrhotic patients with HIV infection.

Keywords: antitubercular agents, drug-induced liver disease, liver cirrhosis, hepatitis, risk factors, tuberculosis

\footnotetext{
*Correspondence to: Roongruedee Chaiteerakij, Department of Medicine, Faculty of Medicine, Chulalongkorn University, Bangkok 10330, Thailand, e-mail: roongruedee.c@chula.ac.th 'Department of Medicine, Faculty of Medicine, Chulalongkorn University and King Chulalongkorn Memorial Hospital, Bangkok 10330, Thailand 2Department of Physiology, Faculty of Medicine, Chulalongkorn University and King Chulalongkorn Memorial Hospital, Bangkok 10330, Thailand ${ }^{3}$ Biostatistics Center, Faculty of Medicine, Chulalongkorn University, Bangkok 10330, Thailand "These authors contributed equally to first authorship of this article. ¿ Open Access. @ 2018 Passisd Laoveeravat et al., published by Sciendo. (c) BY-NC-ND This work is licensed under the Creative Commons Attribution NonCommercial-NoDerivatives 4.0 License.
} 
Tuberculosis and cirrhosis continue to be major public health burdens, particularly in developing countries. Tuberculosis is a leading cause of death worldwide, with 1.5 million deaths attributed to this disease in 2014 alone [1]. Annually, an estimated 9.5 million patients develop symptomatic tuberculosis and 1.4 billion USD is spent for tuberculosis treatment worldwide [1]. There is little research on the impact of tuberculosis in cirrhotic patients; however, patients with cirrhosis have a 3-15-fold greater risk of tuberculosis infection than the general population [2], which is likely due to impaired immunity and malnutrition $[2,3]$.

First-line treatment of tuberculosis includes a combination of the following 4 oral drugs: isoniazid, rifampin, pyrazinamide, and ethambutol. All of these antituberculosis drugs (ATDs), except ethambutol, are considered hepatotoxic, because they can lead to ATD-induced liver injury (ATDILI) $[4,5]$ with higher rates of complication and mortality than other antibiotic agents [6]. Isoniazid and pyrazinamide cause hepatocyte injury by inducing the formation of free radical species, which leads to hepatocyte damage. In addition, isoniazid metabolites (e.g., acetyl hydrazine) are directly toxic to hepatocytes [7]. Differently, rifampin causes cholestasis by competing with enzymes that are used in bilirubin uptake and excretion [5]. All of these 3 drugs are reported to induce liver injury commonly via drug hypersensitivity [5].

Based on the national database in Thailand, cirrhosis and having concomitant disease such as human immunodeficiency virus (HIV) infection are associated with drug-induced liver injury (DILI). Notably, ATDs are 1 of the 2 most common causes of DILI [8]. The incidence of ATDILI in patients without cirrhosis ranges from $8 \%$ to $16 \%$ [9-15] depending on the diagnostic criteria for ATDILI, the study population, and the ATD regimen used. Female sex [9, 10], older age [9, 16, 17], chronic alcohol consumption [14, 15], and chronic viral hepatitis B infection (HBV) $[14,18]$ have all been reported as factors that increase the risk of ATDILI. In addition, elevated baseline serum liver enzyme level [19], total bilirubin (TB) [14], and decreased serum albumin [14, 17] are shown to increase the likelihood of ATDILI.

Individuals with cirrhosis may be at higher risk of developing ATDILI than those without cirrhosis, with incidences of $8 \%-27 \%$ vs. $2 \%-10 \%$ in cirrhotic vs. noncirrhotic patients, respectively [19-21]. A recent study in cirrhotic patients found that $35 \%$ of cirrhotics developed ATDILI, and all these patients had Child-Turcotte-Pugh (CTP) class B or C cirrhosis [22]. Data on risk factors for ATDILI in cirrhotic patients, particularly those with advanced cirrhosis, are limited. Park et al. reported that female sex, increased number of hepatotoxic ATDs, and high baseline alkaline phosphatase (ALP) were factors associated with the development of ATDILI in a cohort of patients with chronic hepatitis and/or early-stage cirrhosis [19]. In this study, only a few patients with end-stage cirrhosis (CTP class C) were included. Another case-control study, by Shin et al., compared the risks of ATDILI in patients with and without cirrhosis [20]. They found that isoniazid and rifampin can be used safely in patients with cirrhosis; however, this study did not include any patients with advanced cirrhosis (CTP class C). Due to the limitations of previous studies, we therefore aimed to identify potential risk factors for ATDILI in cirrhotic patients, particularly patients with advanced-stage cirrhosis.

\section{Materials and methods}

\section{Study patients}

A retrospective cohort study of cirrhotic patients with tuberculosis was conducted at King Chulalongkorn Memorial Hospital, Bangkok, Thailand. This study was approved by the Chulalongkorn University Faculty of Medicine Institutional Review Board (IRB No. 547/58; approval No. 1058/2016) and the study followed the principles of the contemporary revision of the Declaration of Helsinki. The study period was from January 1, 2006, to April 30, 2016. All patients aged 18 years and over and potentially diagnosed with cirrhosis and tuberculosis were identified using the following International Classification of Diseases, tenth revision, Thai Modification (ICD10-TM) codes: A15-A19 for tuberculosis, and K70.3, K74, K76.1 for cirrhosis. The initial inclusion criteria were satisfied in 221 patients. Tuberculosis was defined by positive microbiological tests - either acid-fast stain or culture. Clinical presentation in combination with radiological images including chest x-ray images and/or computed tomography (CT) compatible with tuberculosis was also accepted for the diagnosis of tuberculosis. Cirrhosis was diagnosed by radiological characteristics on abdominal ultrasound, CT, and magnetic resonance (MR) imaging, including small-sized nodular liver and/or caudate lobe hypertrophy or evidence of portal hypertension (collateral vessels, varices, and/or splenomegaly).

\section{Data collection}

Data on demographics, underlying diseases, etiology of cirrhosis, severity of cirrhosis as defined by CTP score/class and model for end-stage liver disease (MELD) score, laboratory tests, and ATD regimen received were abstracted. The ATD regimen prescribed to each individual patient was based on physician's judgment. Other drugs known to be hepatotoxic, e.g., chemotherapy, immunosuppressive agents, or 
antiretroviral drugs, were also abstracted. Because there are currently no definite criteria for the diagnosis of DILI specifically in cirrhotic patients [23-25], the criteria proposed in previous studies were used in this study. The criteria had different cutoffs for patients with normal and abnormal liver enzymes at baseline $[19,26]$. Patients were diagnosed with ATDILI if they met any 1 of the following criteria $[19,26]$ :

(1) Elevated aspartate aminotransferase (AST), alanine transaminase (ALT), or ALP levels $\geq 3$ times of upper limit of normal (ULN) if the baseline level was normal $(n=3)$.

(2) Elevated AST, ALT, or ALP levels $\geq 1.5$ times of the baseline level if the baseline level was elevated $(n=3)$.

Patients who had an elevation of AST, ALT, or ALP due to other identifiable causes, e.g., chronic hepatitis $\mathrm{B}$ and $\mathrm{C}$ infection or septic shock, were not considered as having ATDILI. For patients who had elevated liver enzymes while receiving ATDs and other drugs concomitantly that are potentially hepatotoxic, e.g., antiretroviral drugs, the most recent drug that was initiated or the drug whose dosage was increased was considered as a cause of DILI. Patients with an elevation of liver enzymes within $<3$ days after ATD initiation with or without other events including infection were not considered as having ATDILI.

\section{Statistical analyses}

For baseline characteristics, continuous variables are presented as mean \pm standard deviation (SD) or median and interquartile range (IQR), and categorical variables are shown as percentages. Differences between the ATDILI and non-ATDILI groups were analyzed using a Student $t$ test or Mann-Whitney $U$ test for continuous variables with normal distribution and skewed distribution, respectively, and by a chi-squared or Fisher exact test for categorical variables, as appropriate. Analysis was conducted using IBM SPSS Statistics for Windows (version 22). $P<0.05$ was considered to be significant in the tests of statistical inference.

\section{Results}

An initial medical record review of 221 patients potentially eligible for the study was performed. We excluded 88 patients who had insufficient evidence supporting a diagnosis of cirrhosis, i.e., no radiological imaging results available for review, and 19 patients who lacked sufficient proof for a diagnosis of tuberculosis. The medical records of 114 cirrhotic patients with tuberculosis were subsequently reviewed carefully, and 50 further patients were excluded for a variety of reasons: 7 had died before starting ATDs; 5 had septic shock during the first 30 days after ATD initiation; 5 were given ATD regimen not containing isoniazid, rifampin, or pyrazinamide; 2 were exposed to ATDs for less than 3 days. Another 31 patients who had insufficient information in their medical records were also excluded (Figure 1). The final cohort comprised 64 cirrhotic patients with tuberculosis, of whom 50 and 14 were diagnosed with tuberculosis by microbiological tests and

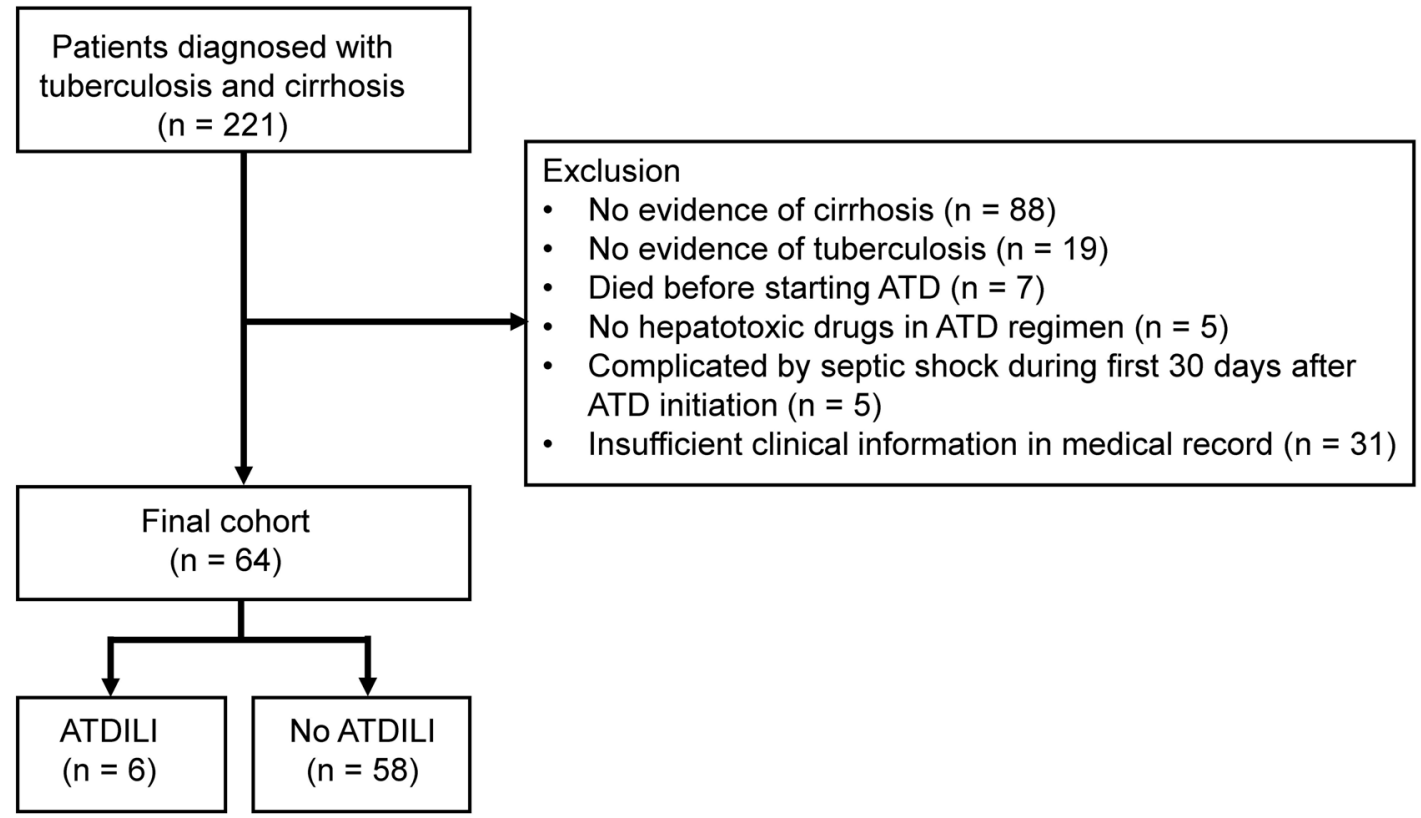

Figure 1. Flowchart of studied patients. ATD, antituberculosis drug; ATDILI, antituberculosis drug-induced liver injury 
combined clinical presentation and radiological imaging, respectively (Table 1).

Of the 64 cirrhotic patients included in the analysis, 45 $(70 \%)$ were male with a mean (SD) age of 54.4 (13.2) years. The diagnosis of cirrhosis was made by the characteristics of cirrhosis and portal hypertension found in either ultrasound $(\mathrm{n}=32,50 \%)$, CT $(\mathrm{n}=19,30 \%)$, or MR $(\mathrm{n}=13,2 \%)$ images. Sixteen (27\%), 32 (53\%), and 12 (20\%) patients had CTP class $\mathrm{A}, \mathrm{B}$, and $\mathrm{C}$ cirrhosis, respectively; 4 patients lacked sufficient information to determine the CTP class. The most common cause of cirrhosis was alcohol $(\mathrm{n}=21,33 \%)$, followed by HBV and viral hepatitis $\mathrm{C}$ infection (HCV) $(n=13,20 \%)$. The most common site of tuberculosis infection was lung and/or pleura $(n=40,64 \%)$, followed by disseminated tuberculosis with 2 or more infected organs $(\mathrm{n}=17,27 \%)$, and extrapulmonary tuberculosis $(\mathrm{n}=6,10 \% ; 1$ patient with missing data on the site of tuberculosis infection). No patients received concomitant cancer chemotherapy or immunosuppressive drugs. Initial ATDs were prescribed to each individual patient based

Table 1. Characteristics of tuberculosis in the study cohort

\begin{tabular}{lc}
\hline Features & $\mathbf{n}(\%)$ \\
\hline Organs of tuberculosis infection $^{\dagger}$ & \\
Pulmonary & $40(64 \%)$ \\
Extrapulmonary & $6(10 \%)$ \\
Disseminated ( $\geq 2$ organs involved) & $17(27 \%)$ \\
Diagnostic methods for tuberculosis & \\
Biopsy & \\
PCR & $12(19 \%)$ \\
AFB smear & $20(31 \%)$ \\
CT imaging & $18(28 \%)$ \\
Chest x-ray imaging & $1(2 \%)$ \\
Clinical diagnosis & $10(16 \%)$ \\
\hline
\end{tabular}

${ }^{\dagger} \mathrm{n}=63 ; 1$ patient had missing data

* Patients who had more than 1 method of diagnosis were classified as biopsy positive, followed by PCR, AFB, CT, and CXR accordingly based on the specificity of the diagnostic test.

AFB, acid-fast bacilli; CT, computed tomography; CXR, chest $x$-ray imaging; $\mathrm{PCR}$, polymerase chain reaction on the judgment of the physician. The ATD regimen comprised 3 hepatotoxic drugs (isoniazid, rifampin, and pyrazinamide) was given to $8 / 16(50 \%)$ of the CTP patients in class A, 22/32 $(69 \%)$ of patients in class B, and 5/12 (42\%) of patients in class $\mathrm{C}$ patients (Table 2). The numbers of cirrhotic patients in CTP classes A, B, and C who received the regimen with 2 hepatotoxic drugs (isoniazid and rifampin) and 1 hepatotoxic drug (either isoniazid or rifampin) are summarized in Table 2.

There were $6(9.4 \%)$ cirrhotic patients who developed ATDILI (Tables 3 and 4). The median duration from the initiation of ATDs to the development of ATDILI was 14 days (range: 6-66). All 6 patients had elevated AST or ALT levels with the median AST/ALT level of 47/24 at baseline and 205/112 when ATDILI was detected. The detailed information for each patient developing ATDILI is summarized in Table 5 . Interestingly, there were more patients with HIV infection in the ATDILI group than in the non-ATDILI group (50\% vs. $9 \%$; $P=0.02)$. In addition, white blood cell count was significantly higher in the ATDILI group, compared with the non-ATDILI group, with median (range) of 12,010 (7,010-23,310)/mL vs. $6,375(1,610-18,900) / \mathrm{mL}(P=0.01)$. All patients who developed ATDILI had CTP class B cirrhosis. However, there

Table 3. Comparison of drug-induced liver injury risk factors for each of Child-Turcotte-Pugh score

\begin{tabular}{clll}
\hline & CTP class A & CTP class B & CTP class C \\
\hline Encephalopathy & None & Grade 1-2 & Grade 3-4 \\
ATDILI, n (\%) & $6(100 \%)$ & 0 & 0 \\
Ascites & None & Mild to moderate & Severe \\
ATDILI, n (\%) & $2(33 \%)$ & $1(17 \%)$ & $3(50 \%)$ \\
Bilirubin, mg/dL & $<2$ & $2-3$ & $>3$ \\
ATDILI, n (\%) & $6(100 \%)$ & 0 & 0 \\
Albumin, g/dL & $>3.5$ & $2.8-3.5$ & $<2.8$ \\
ATDILI, n (\%) & 0 & 0 & $6(100 \%)$ \\
INR & $<1.7$ & $1.7-2.3$ & $>2.3$ \\
ATDILI, n (\%) & $6(100 \%)$ & 0 & 0 \\
\hline
\end{tabular}

ATDILI, antituberculosis drug-induced liver injury; CTP, Child-TurcottePugh; INR, international normalized ratio

Table 2. Initial antituberculosis regimen according to Child-Turcotte-Pugh class

Initial antituberculosis drugs regimen (number of patients)

\begin{tabular}{llll}
\cline { 2 - 4 } & Three hepatotoxic drugs & Two hepatotoxic drugs & One hepatotoxic drug \\
\hline CTP class $A(n=16)$ & IRZE (8) & IRE (7) & IEOS (1) \\
CTP class B $(n=32)$ & IRZE (21), IRZES (1) & IRE (6), IREO (1), IREOS (1), IREL (1) & IE (1) \\
CTP class C $(n=12)$ & IRZE (5) & IRE (5) & IEOS (1), REO (1) \\
Not classified $(n=4)$ & IRZE (2) & IRE (2) & - \\
\hline
\end{tabular}


Table 4. Baseline characteristics of study patients

\begin{tabular}{|c|c|c|c|}
\hline Characteristic & ATDILI $(n=6)$ & non-ATDILI ( $n=58$ ) & $P$ \\
\hline Age (years), mean $\pm S D$ (range) & $54 \pm 13(39-69)$ & $55 \pm 13(25-89)$ & 0.86 \\
\hline Male, $\mathrm{n}(\%)$ & $5(83 \%)$ & $40(69 \%)$ & 0.66 \\
\hline \multicolumn{4}{|l|}{ Cause of cirrhosis, n (\%) } \\
\hline HBV & $0(0 \%)$ & $13(22 \%)$ & 0.33 \\
\hline HCV & $2(33 \%)$ & $11(19 \%)$ & 0.59 \\
\hline Alcohol & $1(17 \%)$ & $20(35 \%)$ & 0.65 \\
\hline \multicolumn{4}{|l|}{ Comorbid conditions, n (\%) } \\
\hline HIV & $3(50 \%)$ & $5(9 \%)$ & 0.02 \\
\hline $\mathrm{DM}$ & $1(17 \%)$ & $17(29 \%)$ & 0.67 \\
\hline CKD and ESRD & $0(0 \%)$ & $8(14 \%)$ & $<0.001$ \\
\hline CTP class, $n(\%)^{\dagger}$ & & & 0.054 \\
\hline A & $0(0 \%)$ & $16(30 \%)$ & \\
\hline B & $6(100 \%)$ & $26(48 \%)$ & \\
\hline C & $0(0 \%)$ & $12(22 \%)$ & \\
\hline CTP score, median (range) $)^{\ddagger}$ & $8(7-9)$ & $7(5-13)$ & 0.66 \\
\hline MELD score, median (range) ${ }^{\S}$ & $12.4(10.2-14.5)$ & $11.7(6.4-29.1)$ & 0.76 \\
\hline Number of hepatotoxic ATDs, n (\%) & & & 0.09 \\
\hline 1 & $0(0 \%)$ & $3(5 \%)$ & \\
\hline 2 & $0(0 \%)$ & $24(41 \%)$ & \\
\hline 3 & $6(100 \%)$ & $31(53 \%)$ & \\
\hline Pyrazinamide in initial ATDs & $6(100 \%)$ & $31(53 \%)$ & 0.04 \\
\hline \multicolumn{4}{|c|}{ Baseline laboratory results, median (range) } \\
\hline Total bilirubin, mg/dL & $1.2(0.4-2.6)$ & $1.3(0.3-7.0)$ & 0.61 \\
\hline AST, U/L & $47(18-67)$ & $44(9-406)$ & 0.54 \\
\hline $\mathrm{ALT}, \mathrm{U} / \mathrm{L}$ & $24(9-41)$ & $23(1-181)$ & 0.55 \\
\hline $\mathrm{ALP}, \mathrm{U} / \mathrm{L}$ & $213(143-362)$ & $131(48-615)$ & 0.13 \\
\hline Albumin, $\mathrm{g} / \mathrm{dL}$ & $2.9(1.9-3.0)$ & $2.9(1.5-4.2)$ & 0.25 \\
\hline Creatinine, $\mathrm{mg} / \mathrm{dL}$ & $0.7(0.3-1.4)$ & $0.8(0.4-8.8)$ & 0.41 \\
\hline Hemoglobin, $\mathrm{g} / \mathrm{dL}$ & $9.0(7.7-9.8)$ & $10.0(7.0-15.2)$ & 0.14 \\
\hline Platelet count $/ \mathrm{mL}$ & $265,000(27,000-334,000)$ & $152,000(64,000-511,000)$ & 0.44 \\
\hline White blood cell count $/ \mathrm{mL}$ & $12,010(7,010-23,310)$ & $6,375(1,610-18,900)$ & 0.01 \\
\hline INR & $1.3(1.2-1.4)$ & $1.3(0.9-2.5)$ & 0.94 \\
\hline
\end{tabular}

${ }^{+} \mathrm{n}=60 ; 4$ patients in the non-ATDILI group had missing data for CTP class.

${ }^{*} \mathrm{n}=58 ; 6$ patients in the non-ATDILI group had missing data.

${ }^{5} \mathrm{n}=50 ; 2$ and 12 patients in the ATDILI and non-ATDILI groups had missing data.

ALP, alkaline phosphatase; ALT, alanine transaminase; AST, aspartate aminotransferase; ATD, antituberculosis drug; ATDILI, antituberculosis druginduced liver injury; CKD, chronic kidney disease; CTP, Child-Turcotte-Pugh; DM, diabetes mellitus; ESRD, end-stage renal disease; HBV, viral hepatitis B infection; $\mathrm{HCV}$, viral hepatitis C infection; HIV, human immunodeficiency virus infection; INR, international normalized ratio; MELD, model for end-stage liver disease; TB, total bilirubin

was no significant difference in the CTP score between the ATDILI group and the non-ATDILI group, with a median (range) of $8(7-9)$ and $7(5-13)$, respectively $(P=0.66)$. All patients $(6 / 6,100 \%)$ in the ATDILI group received ATD regimen containing 3 hepatotoxic drugs (isoniazid, rifampicin, and pyrazinamide), whereas only $31 / 58$ (53\%) patients in the non-ATDILI group received 3 hepatotoxic ATDs and 4 (7\%) and $23(40 \%)$ patients in the non-ATDILI group received 1 and 2 hepatotoxic ATDs, respectively, $P=0.09$.

There were no significant differences in sex, age, etiology of cirrhosis, or median score of CTP and MELD between the 2 groups. Similarly, baseline laboratory results including serum aspartate aminotransferase (AST), alanine transaminase (ALT), tuberculosis, hemoglobin, albumin, and international normalized ratio (INR) were not different between the groups.

Among the 6 patients (4 non-HIV and 2 HIV-infected) who developed ATDILI, the concomitant medications used by the 4 non-HIV patients included insulin Mixtard, tamsulosin, tenofovir, lamivudine, and levothyroxine. These drugs are not considered as hepatotoxic [24]. However, the other 2 HIVinfected patients took nevirapine, stavudine, and efavirenz, which are known to be hepatotoxic. 


\section{Subgroup analysis of cirrhotic patients who received ATD regimen containing 3 hepatotoxic drugs}

Because ATDILI developed exclusively in cirrhotic patients who received 3 hepatotoxic drugs (the first-line ATD regimen), we performed a subgroup analysis of 37 patients who received an ATD regimen containing 3 hepatotoxic drugs (Table 6). Of these, 6 (16\%) patients developed ATDILI. All patients who developed ATDILI had CTP class B cirrhosis. There were more patients with HIV infection in the ATDILI group than the non-ATDILI group $(50 \%$ and $13 \%, P=0.07)$. Other factors were not statistically different between the 2 groups.

\section{Discussion}

The present study sought to determine risk factors for ATDILI in cirrhotic patients. Our finding suggests that the 3 hepatotoxic ATD regimens containing a pyrazinamide increase the risk for ATDILI in patients with cirrhosis. HIV infection and elevated white blood cell count also appear to be associated with an increased risk of developing ATDILI in patients with cirrhosis.

Making an accurate diagnosis of ATDILI in cirrhotics is challenging, because diagnostic criteria for ATDILI specifically for cirrhotic patients have not yet been established. The diagnostic criteria for ATDILI currently stated in most guidelines are based on "normal" liver enzyme levels at baseline $[24,25]$. However, this is not always applicable to cirrhotic patients, because they tend to have alternating and elevated liver enzyme levels. Accordingly, Park et al. recently proposed diagnostic criteria of ATDILI for patients with chronic liver disease and cirrhosis [19]. In brief, ATDILI was diagnosed if liver enzyme levels increased $\geq 3$ times the ULN for cirrhotic patients with normal liver enzyme levels at baseline or if liver enzyme levels increased $\geq 1.5$ times of baseline values for those with elevated liver enzyme levels at baseline.

Overall, the incidence of ATDILI in the present study was $9 \%$ and increased to $16 \%$ in patients who received an initial ATD regimen containing 3 hepatotoxic drugs, i.e., isoniazid, rifampicin, and pyrazinamide. The incidence was concordant with previously reported incidence of $8 \%-27 \%$ among cirrhotics [19-21]. Patients with cirrhosis have a tendency to have liver injury due to impaired baseline liver function. Also, the half-life of ATDs is lengthened in patients with cirrhosis due to a decreased ability to metabolize these drugs [21]. Interestingly, all patients who developed ATDILI in this study had CTP class B cirrhosis, while none of CTP class $\mathrm{C}$ cirrhotics developed ATDILI. This finding is counterintuitive, but can be explained by the concern of physicians 
Table 6. Baseline characteristics of cirrhotics receiving 3 hepatotoxic antituberculosis drugs (isoniazid, rifampin, and pyrazinamide)

\begin{tabular}{|c|c|c|c|}
\hline Characteristics & $\begin{array}{l}\text { ATDILI } \\
(n=6)\end{array}$ & $\begin{array}{c}\text { Non-ATDILI } \\
(n=31)\end{array}$ & $P$ \\
\hline Age (years), mean $\pm S D$ (range) & $54 \pm 13(39-69)$ & $54 \pm 13(25-89)$ & 0.65 \\
\hline Male, $\mathrm{n}(\%)$ & $5(83 \%)$ & $21(68 \%)$ & 0.65 \\
\hline \multicolumn{4}{|l|}{ Cause of cirrhosis, n (\%) } \\
\hline HBV & $0(0 \%)$ & $6(19 \%)$ & 0.24 \\
\hline $\mathrm{HCV}$ & $2(33 \%)$ & $5(16 \%)$ & 0.32 \\
\hline Alcohol & $1(17 \%)$ & $12(39 \%)$ & \\
\hline \multicolumn{4}{|l|}{ Alcohol consumption, $\mathrm{n}(\%)$} \\
\hline \multicolumn{4}{|l|}{ Comorbid conditions, n (\%) } \\
\hline HIV & $3(50 \%)$ & $4(13 \%)$ & 0.07 \\
\hline $\mathrm{DM}$ & $1(17 \%)$ & $12(39 \%)$ & 0.39 \\
\hline CKD and ESRD & $1(13 \%)$ & 7 (23\%) & 0.57 \\
\hline CTP class, n (\%)* & & & 0.12 \\
\hline A & $0(0 \%)$ & $8(28 \%)$ & \\
\hline B & $6(100 \%)$ & $16(55 \%)$ & \\
\hline C & $0(0 \%)$ & $5(17 \%)$ & \\
\hline CTP score, median (range) ${ }^{* *}$ & $8(7-9)$ & $7(5-13)$ & \\
\hline MELD score, median (range) ${ }^{* * *}$ & $12.4(10.2-14.5)$ & $12.4(6.4-23.5)$ & 0.51 \\
\hline \multicolumn{4}{|c|}{ Baseline laboratory results, median (range) } \\
\hline Total bilirubin, mg/dL & $1.2(0.4-2.6)$ & $1.2(0.3-7.0)$ & 0.67 \\
\hline AST, U/L & $47(18-67)$ & $44(12-406)$ & 0.46 \\
\hline $\mathrm{ALT}, \mathrm{U} / \mathrm{L}$ & $24(9-41)$ & $26(1-181)$ & 0.48 \\
\hline$A L P, U / L$ & $213(143-362)$ & $130(48-615)$ & 0.33 \\
\hline White blood cell count $/ \mathrm{mL}$ & $12,010(7,010-23,310)$ & $7,830(3,580-18,900)$ & 0.06 \\
\hline
\end{tabular}

${ }^{*} \mathrm{n}=35 ; 2$ patients in the non-ATDILI group had missing data.

${ }^{* *} \mathrm{n}=34 ; 3$ patients in the non-ATDILI group had missing data.

${ }^{* * *} \mathrm{n}=26 ; 2$ patients in the ATDILI group and 9 patients in the non-ATDILI group had missing data.

ALP, alkaline phosphatase; ALT, alanine transaminase; AST, aspartate aminotransferase; ATD, antituberculosis drug; ATDILI, antituberculosis druginduced liver injury; CKD, chronic kidney disease; CTP, Child-Turcotte-Pugh; DM, diabetes mellitus; ESRD, end-stage renal disease; HBV, viral hepatitis B infection; HCV, viral hepatitis C infection; HIV, human immunodeficiency virus infection; MELD, model for end-stage liver disease

for ATDILI in patients with advanced liver disease, and patients with CTP class $\mathrm{C}$ cirrhosis were likely to receive a lesser number of hepatotoxic ATDs [22]. In the present study, only $42 \%$ of patients with CTP class C cirrhosis received an ATD regimen containing 3 hepatotoxic drugs, while up to $69 \%$ of patients with CTP class B cirrhosis received a regimen with 3 hepatotoxic drugs.

In the present study, ATDILI occurred exclusively in patients who received 3 hepatotoxic ATDs including isoniazid, rifampicin, and pyrazinamide. As consistent with previous studies, increasing the number of hepatotoxic drugs in the ATD regimen was shown to be associated with a greater chance of developing ATDILI $[19,27]$. Park et al. found that adding 1 hepatotoxic drug to the ATD regimen increased ATDILI risk by 2.37 -fold [19]. Steele et al. reported that adding rifampicin to the ATD regimen containing isoniazid significantly increased ATDILI incidence from $1.6 \%$ to $2.6 \%$ [27].

The finding of HIV infection as a potential risk factor for ATDILI in patients with cirrhosis is a new observation. To our knowledge, no previous study has mentioned this finding in cirrhotics. Previous study suggested that HIV infection increased the risk of ATDILI in patients with no underlying chronic liver diseases [28]. The risk is possibly due to an alteration of the oxidative pathway in patients with HIV infection [29]. Another possible cause is concomitant hepatotoxic antiretroviral therapy and other concomitant medications for opportunistic infection (e.g., antifungal agents) [30]. In the present study, of the 3 cirrhotic patients with HIV infection who developed ATDILI, 2 received concurrent antiretrovirals known to be hepatotoxic, i.e., nevirapine, stavudine, and efavirenz. However, the patients had received antiretroviral drugs without changing the regimen for 4 and 16 years before the initiation of ATDs; thus, it was unlikely that antiretroviral drugs caused liver injury. In the main analysis of the data, we found that cirrhotic patients with HIV infection had a higher proportion of those who developed ATDILI, i.e., $50 \%$ vs. $9 \%$. Likewise, the subgroup analysis of patients receiving 3 hepatotoxic drugs found that $4(50 \%)$ of cirrhotic patients 
with HIV infection and $3(13 \%)$ of cirrhotic patients without HIV infection developed ATDLI; however, the difference was not quite significant $(P=0.07)$, probably the result of the small number of patients in the subgroup analysis lacking significant power to detect a difference. This finding suggests that cirrhotic patients with HIV infection may have a higher chance of developing ATDILI than those without HIV infection. Similarly, we found that patients in the ATDILI group had a greater number of white blood cells than those in the non-ATDILI group. The high white blood cell counts are likely a reaction secondary to liver injury rather than a factor that increases the chance of developing ATDILI, e.g., alcoholic hepatitis. No patients with ATDILI were actively drinking alcohol at the time of ATDILI diagnosis; however, 1 patient with cirrhosis caused by alcohol did not have available information on the status of alcohol drinking in their medical record.

The earliest ATDILI occurrence observed in this study was 6 days. To avoid ATDILI-induced liver failure, liver enzyme levels should therefore be monitored within 1 week after ATD initiation [31] and repeated periodically for at least 3 months in all cirrhotic patients. Concordantly, the current American Thoracic Society/Centers for Disease Control and Prevention/Infectious Diseases Society of America Clinical Practice Guidelines state that a liver function test should be conducted every 1-4 weeks for at least 2-3 months after drug initiation [25]. Based on pharmacokinetic studies of ATDs thus far, there has been no evidence supporting that a reduction of rifampin and pyrazinamide dosage would decrease the chance of ATDILI. The standard doses of rifampin and pyrazinamide are 10 and $25 \mathrm{mg} / \mathrm{kg}$, respectively. Doses up to 35 and $60 \mathrm{mg} / \mathrm{kg}$ of rifampin and pyrazinamide, respectively, do not increase the risk of hepatotoxicity, while the effectiveness of tuberculosis treatment might increase $[32,33]$. There were no prior drug pharmacokinetics or dose comparison studies in patients with cirrhosis. There are limited data for the dosage of isoniazid and its effect.

A strength of this study was that we were able to identify factors associated with ATDILI specifically for cirrhotic patients, because the study cohort comprised cirrhotic patients exclusively, while the cohorts in previous studies included patients with chronic liver diseases both without cirrhosis and with cirrhosis and other studies included cirrhotic and noncirrhotic patients as cases and controls, respectively [19, 20]. However, the present study also has some limitations. First, due to the retrospective nature of the study, laboratory blood testing schedules varied from physician to physician. As a result, some patients who had asymptomatic liver injury might have been underdetected. Second, the ATD regimen given to each individual patient was prescribed primarily based on the decision of the physicians. The risk of developing ATDILI might therefore have varied, depending on the initial regimen. To address this limitation, we conducted subgroup analysis of cirrhotics with 3 hepatotoxic ATDs and shown the similar trend of increased ATDILI risk in HIVinfected patients. Third, this study was conducted in a national tertiary care hospital. Thus, patients at our center tended to have more complicated underlying conditions, which may increase the chance of ATDILI. Caution should be exercised when applying our results in the context of general hospitals. A relatively large number of patients were excluded from the study cohort due to unavailable imaging to confirm the diagnosis of cirrhosis. Given the small numbers of patients included in this study, a future prospective, multicenter study is warranted to validate these results and determine its generalizability.

\section{Conclusions}

ATDILI commonly occurs in cirrhotic patients. Factors potentially associated with ATDILI included the use of all the 3 hepatotoxic drugs, particularly pyrazinamide, in the initial antituberculosis regimen and HIV infection. To reduce the hepatotoxicity of ATDs, these factors should be considered when prescribing an antituberculosis regimen for cirrhotics. Further study with larger cohorts is warranted to validate the results in this study.

Author contributions. PL, NW, ST, RR, and RC contributed substantially to the concept and design of the study. PL and $\mathrm{NW}$ contributed substantially to the collection of data, and CP, $\mathrm{CH}$, and $\mathrm{RC}$ made substantial contributions to its analysis and interpretation. $\mathrm{CP}$ and $\mathrm{CH}$ conducted the statistical analysis. $\mathrm{PL}, \mathrm{NW}, \mathrm{CP}, \mathrm{CH}$, and RC drafted the manuscript, and ST, RR, and RC contributed to critical revision. ST, RR, and RC provided expertise on DILI. All the authors reviewed and approved the final version of the manuscript submitted for publication and take responsibility for statements made in the published article.

Acknowledgments. The authors gratefully acknowledge the contributions of Kevin P. Jones for language editing and Pornthip Sinthavanuruk for administrative assistance. The present study was presented as poster presentation at the 2016 Digestive Disease Week, May 21-24, 2016, San Diego, California, Laoveeravat P, Wongjarupong N, Phathong C, Rerknimitr R, Chaiteerakij R. Risk factors and a predictive score for anti-tuberculosis drug-induced livery injury (at-DILI) in patients with cirrhosis [Sa1562]. 
Gastroenterology. 2016; 150(4), S1067-8 (American Association for the Study of Liver Disease (AASLD) abstracts). We did not receive any specific grant for this research from any funding agency in the public, commercial, or not-forprofit sectors.

Conflict of interest statement. The authors have each completed and submitted an International Committee of Medical Journal Editors Uniform Disclosure Form for Potential Conflicts of Interest. None of the authors have any potential conflict of interest to disclose.

\section{References}

[1] World Health Organization. Global tuberculosis report 2015. Geneva: WHO Press; 2015.

[2] Thulstrup AM, Mølle I, Svendsen N, Sørensen HT. Incidence and prognosis of tuberculosis in patients with cirrhosis of the liver. A Danish nationwide population based study. Epidemiol Infect. 2000; 124:221-5.

[3] Kumar N, Kedarisetty CK, Kumar S, Khillan V, Sarin SK. Antitubercular therapy in patients with cirrhosis: Challenges and options. World J Gastroenterol. 2014; 20:5760-72.

[4] Navarro VJ, Senior JR. Drug-related hepatotoxicity. N Engl J Med. 2006; 354:731-9.

[5] Senousy BE, Belal SI, Draganov PV. Hepatotoxic effects of therapies for tuberculosis. Nat Rev Gastroenterol Hepatol. 2010; 7:543-56.

[6] Treeprasertsuk S, Huntrakul J, Ridtitid W, Kullavanijaya P, Björnsson ES. The predictors of complications in patients with drug-induced liver injury caused by antimicrobial agents. Aliment Pharmacol Ther. 2010; 31:1200-7.

[7] Perwitasari DA, Atthobari J, Wilffert B. Pharmacogenetics of isoniazid-induced hepatotoxicity. Drug Metab Rev. 2015; 47:222-8.

[8] Sobhonslidsuk A, Poovorawan K, Soonthornworasiri N, Pan-ngum W, Phaosawasdi K. The incidence, presentation, outcomes, risk of mortality and economic data of drug-induced liver injury from a national database in Thailand: a population-base study. BMC Gastroenterol. 2016; 16:135. doi:10.1186/s12876-016-0550-0.

[9] Dossing M, Wilcke JT, Askgaard DS, Nybo B. Liver injury during antituberculosis treatment: an 11-year study. Tuber Lung Dis. 1996; 77:335-40.

[10] Golemba AS, Ferreyra FG, Martearena RE, Achinelli FR, Rovai GB. Drug-induced hepatotoxicity and tuberculosis in a hospital from the Argentinian northeast: cross-sectional study. Medwave. 2015; 15:e6135. doi:10.5867/medwave.2015.04.6135.

[11] Gülbay BE, Gürkan OU, Yıldız OA, Önen ZP, Erkekol FO, Baççıŏlu A, Acıcan T. Side effects due to primary antituberculosis drugs during the initial phase of therapy in 1149 hospitalized patients for tuberculosis. Respir Med. 2006; 100:1834-42.

[12] Isa SE, Ebonyi AO, Shehu NY, Idoko P, Anejo-Okopi JA, Simji G, et al. Antituberculosis drugs and hepatotoxicity among hospitalized patients in Jos, Nigeria. Int J Mycobacteriol. 2016; 5:21-6.

[13] Saha A, Shanthi FXM, Winston AB, Das S, Kumar A, Michael JS, Balamugesh T. Prevalence of hepatotoxicity from antituberculosis therapy: a five-year experience from South India. J Prim Care Community Health. 2016; 7:171-4.

[14] Sun Q, Zhang Q, Gu J, Sun W-W, Wang P, Bai C, et al. Prevalence, risk factors, management, and treatment outcomes of first-line antituberculous drug-induced liver injury: a prospective cohort study. Pharmacoepidemiol Drug Saf. 2016; 25:908-17.

[15] Wondwossen A, Waqtola C, Gemeda A. Incidence of antituberculosis-drug-induced hepatotoxicity and associated risk factors among tuberculosis patients in Dawro Zone, South Ethiopia: a cohort study. Int J Mycobacteriol. 2016; 5:14-20.

[16] Hosford JD, von Fricken ME, Lauzardo M, Chang M, Dai Y, Lyon JA, et al. Hepatotoxicity from antituberculous therapy in the elderly: a systematic review. Tuberculosis. 2015; 95:112-22.

[17] Singla R, Sharma SK, Mohan A, Makharia G, Sreenivas V, Jha B, et al. Evaluation of risk factors for antituberculosis treatment induced hepatotoxicity. Indian J Med Res. 2010; 132:81-6.

[18] Wang N-T, Huang Y-S, Lin M-H, Huang B, Perng C-L, Lin H-C. Chronic hepatitis $\mathrm{B}$ infection and risk of antituberculosis drug-induced liver injury: systematic review and meta-analysis. J Chin Med Assoc. 2016; 79:368-74.

[19] Park WB, Kim W, Lee KL, Yim J-J, Kim M, Jung YJ, et al. Antituberculosis drug-induced liver injury in chronic hepatitis and cirrhosis. J Infect. 2010; 61:323-9.

[20] Shin HJ, Lee HS, Kim YI, Lim SC, Jung JP, Ko YC, Kwon YS. Hepatotoxicity of anti-tuberculosis chemotherapy in patients with liver cirrhosis. Int J Tuberc Lung Dis. 2014; 18:347-51.

[21] Cho Y-J, Lee SM, Yoo C-G, Kim YW, Han SK, Shim Y-S, Yim J-J. Clinical characteristics of tuberculosis in patients with liver cirrhosis. Respirology. 2007; 12:401-5.

[22] Sharma P, Tyagi P, Singla V, Bansal N, Kumar A, Arora A. Clinical and biochemical profile of tuberculosis in patients with liver cirrhosis. J Clin Exp Hepatol. 2015; 5:8-13.

[23] Aithal GP, Watkins PB, Andrade RJ, Larrey D, Molokhia M, Takikawa $\mathrm{H}$, et al. Case definition and phenotype standardization in drug-induced liver injury. Clin Pharmacol Ther. 2011; 89:806-15.

[24] Fontana RJ, Watkins PB, Bonkovsky HL, Chalasani N, Davern T, Serrano J, Rochon J; DILIN Study Group. Drug Induced Liver Injury Network (DILIN) prospective study: rationale, design and conduct. Drug Saf. 2009; 32:55-68. 20

[25] Nahid P, Dorman SE, Alipanah N, Barry PM, Brozek JL, Cattamanchi A, et al. Official American Thoracic Society/Centers for Disease Control and Prevention/Infectious Diseases Society of America Clinical Practice Guidelines: treatment of drug-susceptible tuberculosis. Clin Infect Dis. 2016; 63:e147-95.

[26] Wong W-M, Wu P-C, Yuen M-F, Cheng C-C, Yew W-W, Wong P-C, et al. Antituberculosis drug-related liver dysfunction in chronic hepatitis B infection. Hepatology. 2000; 31:201-6.

[27] Steele MA, Burk RF, DesPrez RM. Toxic hepatitis with isoniazid and rifampin: a meta-analysis. Chest. 1991; 99:465-71.

[28] Yimer G, Gry M, Amogne W, Makonnen E, Habtewold A, Petros $Z$, et al. Evaluation of patterns of liver toxicity in patients on antiretroviral and anti-tuberculosis drugs: a prospective four arm observational study in Ethiopian patients. PloS ONE. 2014; 9:e94271. doi:10.1371/journal.pone.0094271.

[29] Lee BL, Wong D, Benowitz NL, Sullam PM. Altered patterns of drug metabolism in patients with acquired immunodeficiency syndrome. Clin Pharmacol Ther. 1993; 53:529-35. 
[30] Naidoo S, Evans D, Jong E, Mellet K, Berhanu R. Outcomes of TB/HIV co-infected patients presenting with antituberculosis drug-induced liver injury. S Afr Med J. 2015; 105:393-6.

[31] Dhiman RK, Saraswat VA, Rajekar H, Reddy C, Chawla YK. A guide to the management of tuberculosis in patients with chronic liver disease. J Clin Exp Hepatol. 2012; $2: 260-70$.
[32] Boeree, MJ, Diacon AH, Dawson R, Narunsky K, du Bois J, Venter A, et al. A dose-ranging trial to optimize the dose of rifampin in the treatment of tuberculosis. Am J Respir Crit Care Med. 2015; 191:1058-65.

[33] Pasipanodya, JG, Gumbo T. Clinical and toxicodynamic evidence that high-dose pyrazinamide is not more hepatotoxic than the low doses currently used. Antimicrob Agents Chemother. 2010; 54:2847-54. 